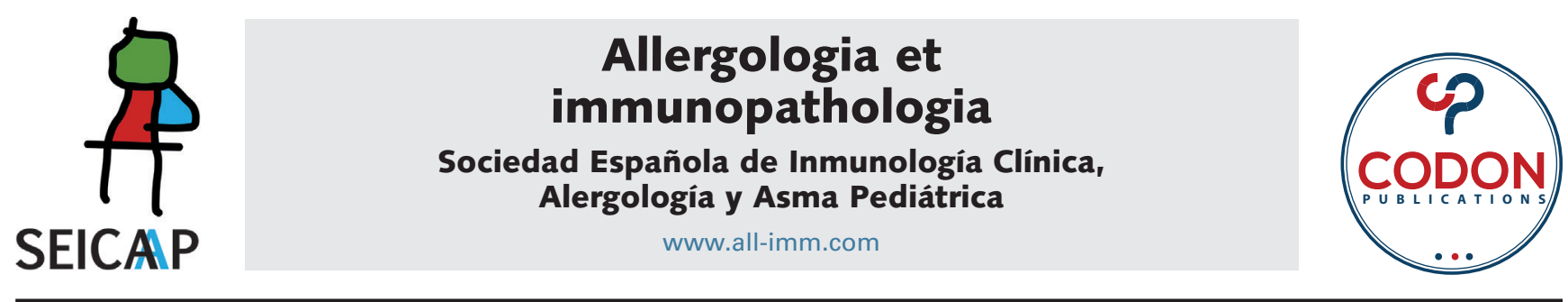

ORIGINAL ARTICLE

OPEN ACCESS (c) $\underset{\mathrm{BY}}{\mathrm{NC}(\mathrm{FA}}$

\title{
Perturbation of monocyte subsets in iron-deficient children - a shift to a pro-inflammatory state?
}

\author{
Nimisha Dhankara, Richa Gupta ${ }^{\mathrm{b} *}$, Shyam Lata Jainc, Shramana Mandal ${ }^{\mathrm{d}}$, Beauty Sarkar
}

a-d Maulana Azad Medical College, New Delhi, India

eUniversity College of Medical Science and Guru Teg Bahadur Hospital, New Delhi, India

Received 25 November 2020; Accepted 25 June 2021

Available online 1 November 2021

\section{KEYWORDS \\ Iron Deficiency \\ anemia; \\ Children; \\ Monocyte subsets; \\ Flow cytometry}

\begin{abstract}
Background: Iron deficiency anemia (IDA) is the most prevalent micronutrient deficiency in preschool children in developing countries including India. IDA is associated with immune perturbation, which is reflected in greater frequency of infections in these children. Recent research has shown three distinct monocyte subsets with distinct functions linked to infectious, inflammatory, and autoimmune diseases. These subsets have not been studied in children with IDA.

Objective: The aim of the study was to assess the percentage of monocyte population and the three subset populations in children with IDA and to compare the data with age-matched healthy controls.

Methods: Venous blood samples $(5 \mathrm{~mL})$ from 40 IDA children and 20 controls were collected after taking informed consent. Monocyte subpopulations were compared across the two groups. The outcome variables were calculated using Students Independent $t$-test or MannWhitney $U$ test. $P$ value of $<0.05$ was taken as significant.

Results: No significant difference was found in the absolute numbers as well as percentages of total monocytes between the control and case (study) group. Children in the IDA group showed a significant $(p=0.03)$ decrease in the nonclassical subset population when compared to the control group.

Conclusion: This is the first study done on monocyte subsets in iron-deficient children. Decrease in nonclassical monocytes observed may be associated with a pro-inflammatory state and increased risk of inflammatory and auto immune diseases. Follow-up studies are needed to confirm these findings.

(c) 2021 Codon Publications. Published by Codon Publications.
\end{abstract}

*Corresponding author: Richa Gupta, College block, University College of Medical Sciences and GTB Hospital, Delhi. Email address: richagupta0209@gmail.com 


\section{Introduction}

Iron Deficiency Anemia (IDA) is one of the most prevalent micronutrient deficiencies in children, especially in developing countries. The World Health Organization (WHO) reported a $59 \%$ prevalence of anemia among Indian preschool children, iron deficiency being the most common. ${ }^{1}$ Iron deficiency has manifold adverse impacts on health including a weakened immune system. ${ }^{2,3}$

Various studies have shown that Iron deficiency is associated with defects in B- and T-cell functions. ${ }^{4}$ However, the effect of iron deficiency on the innate immune system is not well elaborated. Some studies have shown that iron deficiency is associated with decreased radical oxygen species formation, decreased oxidative burst, and reduced production of macrophage migration inhibition factor by phagocytic cells. ${ }^{5-7}$ Monocytes are the primary phagocytic cells of the body and comprise around 5\%-10\% of the white blood cells in humans. Recently, three different subsets of monocytes have been described in humans based on differential flow cytometric expression of CD14 (co-receptor for toll-like receptor 4 (TLR4) and CD16 (FC gamma receptor IIla). ${ }^{8,9}$ The classical subset - comprising majority of circulating monocytes with high phagocytic and antimicrobial activity; nonclassical subset - comprising $2 \%-11 \%$ of circulating monocytes; and the intermediate subset - comprising a small and variable percentage. These subsets differ in their functional characteristics and perturbations in the relative percentage of different subsets and can lead to a lack of ability to mount an adequate immune response against various microbes or a pro-inflammatory state. Disturbance in the balance of the three subsets has been linked with the pathogenesis of diseases like diabetes, obesity, cardiovascular illness, as well as auto-immunity. ${ }^{10,11}$ However, studies to assess the direct impact of IDA on monocyte subset populations are lacking. Thus, the aim of this study was to assess the percentage of monocyte population and the three subset populations in children with IDA and to compare the data with agematched healthy controls.

\section{Materials and methods}

This was an observational, cross-sectional study. Children between 1 and 12 years of age presenting to the Pediatrics OPD of a tertiary care center were enrolled for the study. Ethical clearance for the study was obtained from Institutional ethics committee. A total of 40 cases and 20 controls were selected after applying stringent inclusion and exclusion criteria. Written informed consent was taken from all subjects.

\section{Inclusion criteria included}

Hemoglobin $(\mathrm{Hb})$ levels below two standard deviations for age, mean corpuscular volume (MCV) less than 80 femtoliters $(\mathrm{fL})$, serum ferritin levels below $7 \mathrm{ng} / \mathrm{mL}$, and/or transferrin saturation less than $10 \%$. Children with albumin levels $<3.5 \mathrm{~g} / \mathrm{dl}$, history of recent acute blood loss, dimorphic anemia, severe acute malnutrition, history of receiving iron replacement therapy or other hematinics in the last 3 months, history of intake of immunosuppressant drugs, radiotherapy, or chemotherapy in the last 1 year were excluded from the study. Diagnosed cases of autoimmune diseases, chronic illness (renal, hepatic illness), or malignancy were also excluded. Children in the control group were healthy, matched for age, and had normal $\mathrm{Hb}$, MCV, and hematocrit levels for age.

\section{Sampling}

Venous blood samples were collected from both cases and controls under complete aseptic conditions. Blood $(2 \mathrm{~mL})$ was withdrawn in K3-EDTA anticoagulant tubes for complete blood counts $(C B C)$, peripheral blood smear (PBS) examination, and flow cytometric analysis. In addition, $3 \mathrm{~mL}$ of blood was collected in a plain tube (no anticoagulant), left to clot, and centrifuged at $5000 \mathrm{rpm}$ for $5 \mathrm{~min}$. The serum was then separated and stored at $-20^{\circ} \mathrm{C}$ for iron studies and albumin quantification.

\section{Laboratory analysis}

CBC was performed on automated hematology analyzer (Sysmex XT-1800i). Hb, total leucocyte count (TLC), mean corpuscular volume (MCV), hematocrit, platelet count, and other RBC parameters were assessed. PBS was made according to the standard guidelines using Giemsa stain. Flow cytometric analysis was performed on FC500 Beckman and Coulter dual laser, five color instrument. Gating strategy CD45 versus SS (side scatter) was used to separate lymphocytes and monocytes. Monocytes were further gated on the basis of expression of CD11b and HLA DR. On the basis of differential staining of CD14 and CD16, they were divided into three subpopulations [classical (CD14+/ CD16-), nonclassical (low CD14+/CD16+), and intermediate CD14+/CD16+)] (Figure 1).

Biochemical analysis was performed on a fully automated clinical chemistry analyzer (Randox Imola) in the Department of Biochemistry: Serum ferritin (ng/dl) was measured by electro-chemiluminescence; serum albumin $(\mathrm{g} / \mathrm{dl})$, serum iron $(\mathrm{mcg} / \mathrm{dl})$, and TIBC $(\mathrm{mcg} / \mathrm{dl})$ were measured by spectrophotometry. Transferrin saturation (\%) was calculated using the formula: serum iron/total iron binding capacity $\times 100$.

\section{Statistical analysis}

Statistical analysis was performed using SPSS software version 17. The outcome variables were expressed in means and medians and the difference between the mean and median serum values of all the outcome variables was calculated using Students Independent $t$-test or Mann-Whitney $U$ test. $P$ value of $<0.05$ was taken as significant. Linear regression analysis was done using the Spearman correlation test. 

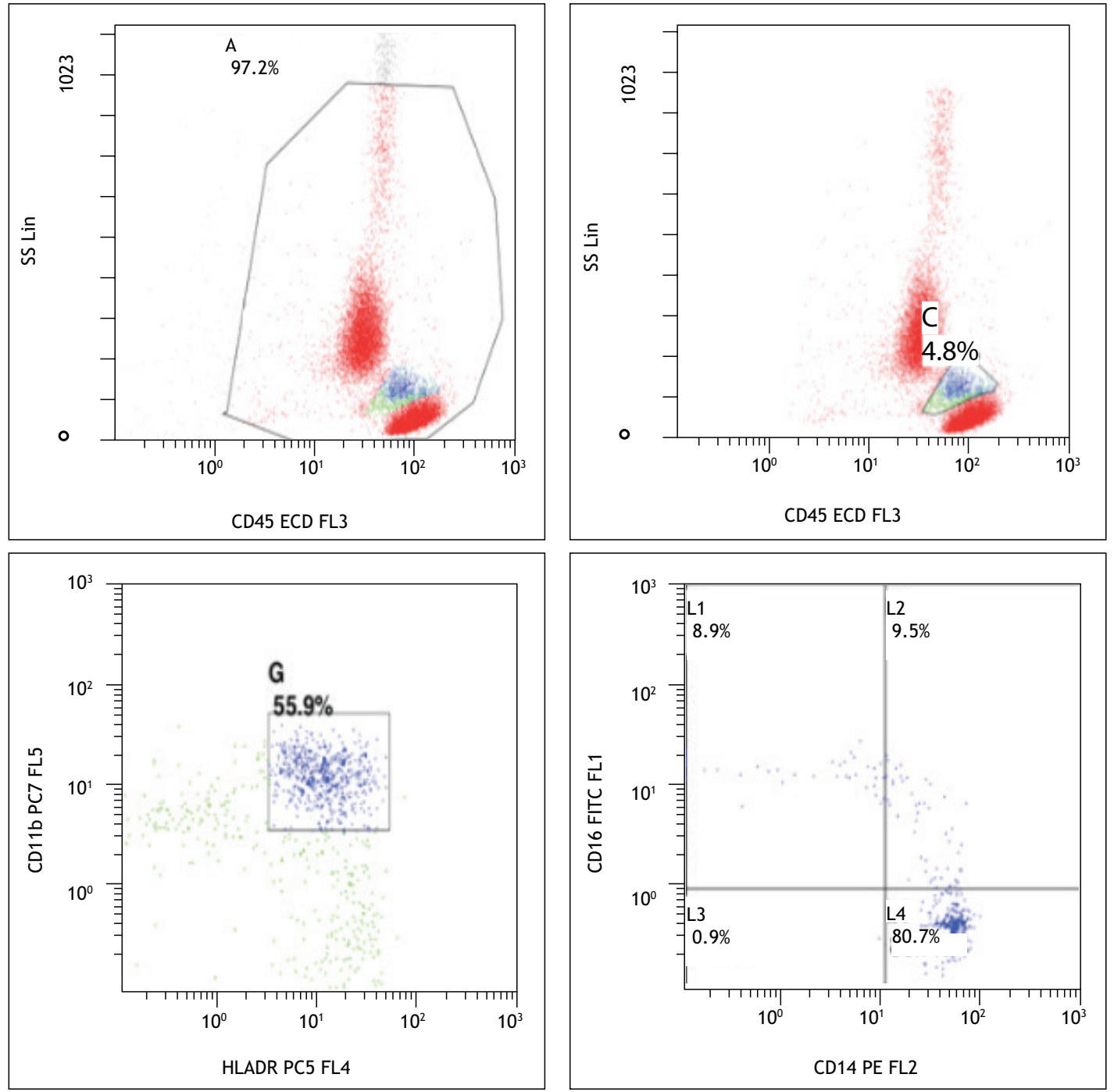

Figure 1 Monocyte gating strategy and separation of monocyte subsets (control).

\section{Results}

The two groups were matched for age. Pallor, atrophic glossitis, and pica were present in $100 \%, 27.5 \%$, and $20 \%$ of the cases (study group), respectively. None of the subjects in the control group had any of these clinical features. There was a significant difference in the $\mathrm{Hb}$ and MCV values in the two groups $(p<0.0001$ for both). The cases showed a decrease in the mean and median $\mathrm{Hb}$ $(8.09 \mathrm{gm} / \mathrm{dl}, 8.5 \mathrm{gm} / \mathrm{dl}$ resp.) as compared to the control group $(12.25 \mathrm{gm} / \mathrm{dl}, 12.35 \mathrm{gm} / \mathrm{dl})$. Serum iron levels, ferritin levels, and transferrin saturation showed a significant decrease in the case group when compared to the control group $(p<0.0001)$. Serum TIBC showed statistically significant increase in the case group when compared to the control group $(p<0.0001)$, as expected. On the other hand, serum albumin levels were comparable in the two groups $(p=0.27)$ (Table 1).

The total leucocyte count (TLC), although within normal limits, was found to be significantly lower in the IDA group as compared to the control group $(p=0.03)$ with mean TLC being 9261.5 and 8387.7, respectively. However, the percentage of total lymphocytes as well as B lymphocytes was significantly higher in the IDA group as compared to the controls ( $p$ of 0.04 for both). The difference regarding the percentage of $T$ lymphocytes $(p=0.07)$ was not significant (Table 2).

No significant difference was found in the absolute numbers as well as percentages of total monocytes between the control and case group. Children in the IDA group showed a significant $(p=0.03)$ decrease in the nonclassical subset population when compared to the control group (Figure 2).

The percentage of intermediate and classical monocytes was found to be increased in the cases; however, the difference was not significant (Table 2). Spearman test was applied to assess the correlation between the percentage of nonclassical subset and hemoglobin and iron parameters (S. Iron, S. Ferritin, TIBC, and Transferrin saturation). It revealed no significant correlation. Spearman correlation test was applied to assess the correlation between the percentage of nonclassical monocyte and total lymphocytes, 
B lymphocytes, and T lymphocytes, and it found no significant correlation as well.

\section{Discussion}

The present study found a significant decrease in the nonclassical subset in the IDA group as compared to the control

Table 1 Iron indices in the IDA and control group.

\begin{tabular}{lccc}
\hline Parameter & $\begin{array}{c}\text { Control group } \\
(\mathrm{n}=20)\end{array}$ & $\begin{array}{c}\text { Case group } \\
(\mathrm{n}=40)\end{array}$ & P value \\
\hline Age & $6.2 \pm 2.3$ & $5.31 \pm 3.12$ & 0.16 \\
$\mathrm{Hb}$ & $12.25 \pm 0.62$ & $8.09 \pm 1.86$ & $<0.0001$ \\
S. Ferritin & $78.7 \pm 18.71$ & $5.27 \pm 1.08$ & $<\mathbf{0 . 0 0 0 1}$ \\
S. Iron & $88 \pm 19.84$ & $24.63 \pm 7.7$ & $<\mathbf{0 . 0 0 0 1}$ \\
S. TIBC & $276.72 \pm 46.65$ & $410.24 \pm 64.22$ & $<\mathbf{0 . 0 0 0 1}$ \\
Transferrin Sat. & $32.44 \pm 8.19$ & $6.25 \pm 2.15$ & $<\mathbf{0 . 0 0 0 1}$ \\
\hline
\end{tabular}
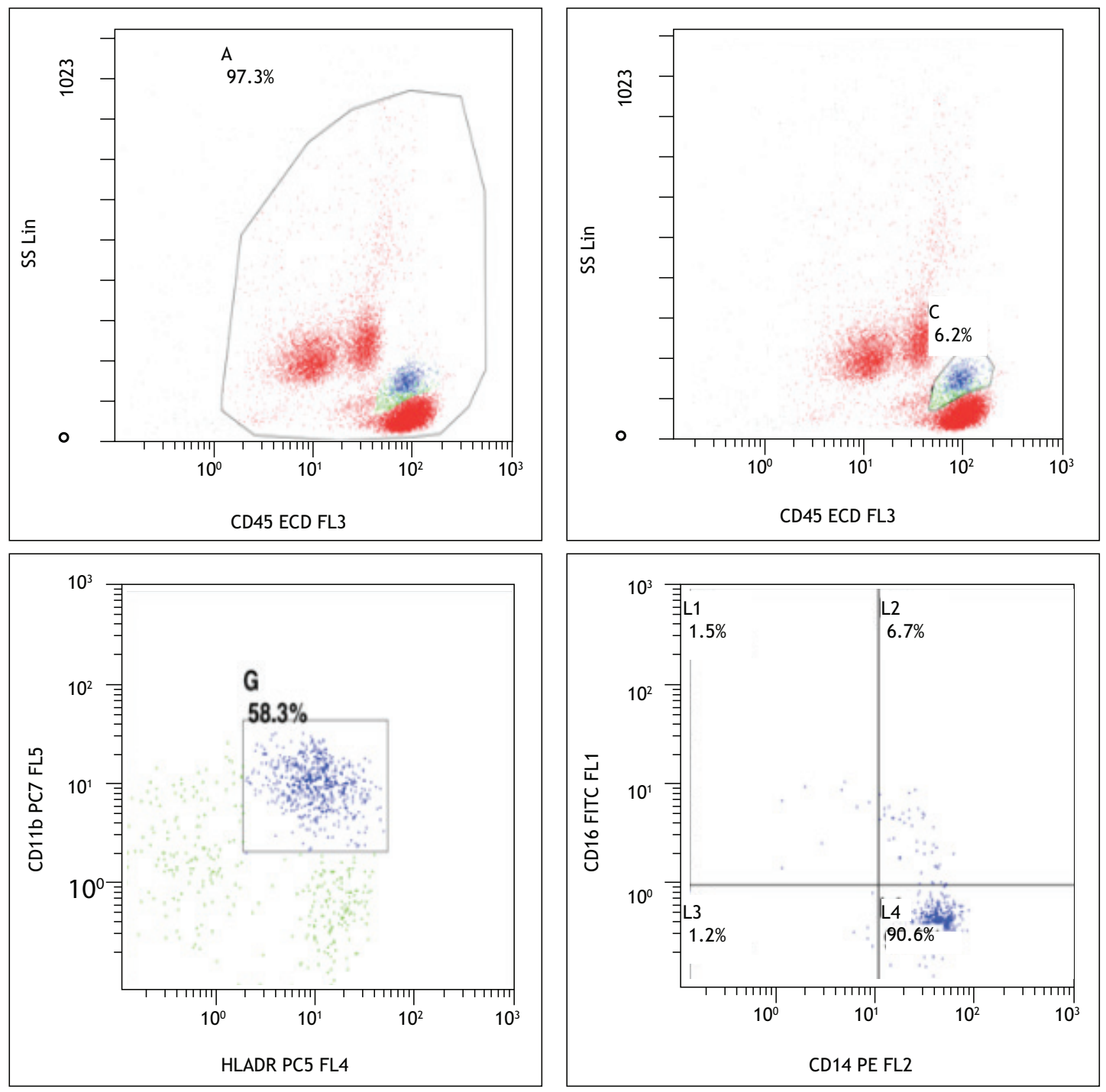

Figure $2-7 y / M$ with iron deficiency showing decrease in nonclassical monocytes. 
group $(p=0.033)$. There was a concomitant increase in the classical and intermediate subsets; however, it was not significant ( $p=0.34,0.90$, respectively).

The study found no significant alteration in the percentages and absolute number of monocytes in iron deficiency as compared to healthy population $(p=0.74,0.75$, respectively). Sadeghian et al. conducted a study in 90 premenopausal women and found that relative count of monocytes in the IDA group was significantly more than the control group. ${ }^{12}$ In contrast, a study by Ahluwalia et al. on 72 homebound elderly women found total monocyte numbers and percentages showing no significant difference between the iron-deficient and healthy group. ${ }^{13}$ This is in concordance with our findings. There are very few studies comparing the monocyte numbers in healthy and iron-deficient children. Since monocytes are one of the most important cellular components of the innate immune response, a normal number of monocytes implies that subjects with iron deficiency may be capable of mounting an adequate phagocytic response to infections. However, Berrak et al. tried to evaluate the effect of iron deficiency on neutrophilic and monocytic apoptotic response in 49 iron-deficient children. ${ }^{14}$ They found an altered response in ID which was partially corrected after iron supplementation. They concluded ID is associated with functional defects in monocytes. Another study by Ekiz et al. demonstrated that phagocytic activity of monocytes was significantly decreased in patients who had IDA. ${ }^{15}$ Thus, although the monocyte numbers may not be altered, a functional defect may be seen in monocytes in association with iron deficiency. The functional defect was however not evaluated in the present study.

With the advent of flow cytometry, three phenotypically and functionally distinct monocyte populations have been described:

1. Classical monocytes: They comprise about $80 \%-95 \%$ of the circulating monocytes and express high CD14 and no CD16. They have high phagocytic and antimicrobial activity along with pro-inflammatory activity.

2. Intermediate monocytes: They express both CD14 and CD16 and comprise $2 \%-8 \%$ of the total circulating monocyte population. They appear to be a transitional population and have both inflammatory and phagocytic functions. They are involved in production of ROS, antigen presentation, and stimulation of T cells. Their role in angiogenesis has also been well documented.

3. Nonclassical monocytes: They comprise $2 \%-11 \%$ of circulating monocytes and express low CD14 and high CD16. They are described to be mobile in nature and patrol the endothelium in search of injury and removal of debris. They are also involved in antigen presentation and T-cell stimulation besides having pro apoptotic and antiproliferative activity. ${ }^{16}$

This study found a significant decrease in the nonclassical subset in iron-deficient children. Nonclassical monocytes have been widely viewed as anti-inflammatory and important for the maintenance of vascular homeostasis. They are a first line of defense in recognition and clearance of pathogens and thus play a protective role by phagocytosis of pathogens and debris immediately under the endothelium. This subset has also been found to be positively associated with disease burden, especially in chronic diseases. Mukherjee et al. analyzed the monocyte subsets in patients with sepsis and systemic lupus erythematosus (SLE). ${ }^{16}$ They found that both intermediate and nonclassical subsets were increased in patients of sepsis with concomitant decrease in the classical subtype. Following treatment, the nonclassical subset returned back to normal. In patients of SLE, only the nonclassical subset showed an increase. It was postulated that the intermediate subset is only increased in chronic conditions, while nonclassical monocytes are increased in both acute and chronic inflammatory conditions. The nonclassical monocytes are also found to be increased in tuberculosis and are corrected after initiation of antitubercular treatment. The decrease in this protective subset of monocytes in iron-deficient children may contribute to increased susceptibility of these patients to infection as well as a pro-inflammatory state which may further compound the anemia by disturbing homeostasis.

The study also found an increase in the intermediate monocyte subsets in the iron-deficient children, although it was not statistically significant. Their exact role is elusive, but intermediate monocytes produce both pro- and antiinflammatory mediators simultaneously. They also produce high levels of reactive oxygen species along with enhanced phagocytic potential. During homeostasis, the intermediate subsets have the highest expression of antigen-presenting molecules. ${ }^{17}$ Many studies showed that the intermediate monocytes are present in the blood of patients with systemic infection. A report by Leodore et al. suggested that in chronic conditions like COPD, there is over expression of intermediate monocytes and decrease in nonclassical subset. ${ }^{18}$ An intermediate subset has also been shown to expand in many inflammatory and autoimmune conditions including chronic kidney disease, active rheumatoid arthritis, coronary artery disease, and type 2 diabetes mellitus. In a study by Eljaszewicz et al., the increase in the intermediate subset was associated with an increased risk of cardiovascular event in CRF patients. ${ }^{19}$ Ren et al. studied the intermediate monocytes in 54 children with recent onset type 1 diabetes mellitus and compared them with healthy controls. Their study showed a significant increase in intermediate monocytes in children with type 1 diabetes mellitus as compared to controls. ${ }^{20}$ The authors suggested that expanded intermediate monocytes are a predictive factor for the poor residual islet $\beta$-cell function in these children as these cells may be responsible for enhanced production of proinflammatory cytokines. Thus, it may be hypothesized that an increase in the intermediate monocytes in iron deficiency leads to alteration of the immune milieu and may be associated with the induction of an enhanced inflammatory immune response in these children making them susceptible to diseases linked to inflammation and auto immunity.

In the current study, an increase in the classical subset of monocytes was seen in children with IDA when compared with healthy controls. The classical monocytes are the most prevalent subset in peripheral blood. Classical monocytes are rapidly recruited to the site of inflammation where they mediate in the inflammatory process leading to pro-inflammatory phenomenon via their ability to 
secrete soluble mediators and to differentiate into monocyte-derived dendritic cells to bridge innate and adaptive immune responses and phagocytosis. ${ }^{17}$ They are known to be important scavenger cells. The classical subset being pro-inflammatory in nature may promote a chronic inflammatory state in these individuals. However, looking at the cytokine milieu, more studies are required to arrive at a conclusive opinion. Unfortunately, the serum level of these cytokines was not investigated in this current study.

Various in vitro studies and review articles have concluded that IDA is associated with decreased T-cell proliferation. Since nonclassical subset is involved in T-cell stimulation, it might contribute to the decreased T-cell proliferation found in IDA. ${ }^{4,21}$ However, decreased T-cell proliferation was not reflected in our study. Although a quantitative defect in T-cells was ruled out, the presence of a qualitative defect in T-cells needs to be further investigated. No correlation was seen between different monocyte subsets and effector and/or cytotoxic T-cells. The study by Ren et al. in diabetic children demonstrated that the increase in intermediate monocytes was positively correlated with increase in memory T-cells in these children. ${ }^{20}$ This subset was not separately studied due to economic constraints.

To the best of our knowledge, this is the first study evaluating monocyte subsets in IDA in children. The limitation of the study was a small sample size due to which the correlation of subset perturbation and severity of anemia could not be established. Further studies with larger sample size are needed to arrive at a conclusive diagnosis.

\section{Conclusion}

IDA was found to be associated with a significant decrease in the nonclassical monocytes and an increase in intermediate and classical monocytes in these children. Together, this perturbation induces a pro-inflammatory state which may make these children vulnerable to inflammatory and autoimmune diseases. However, larger studies are needed to confirm these findings.

\section{References}

1. World Health Organization. The global prevalence of anaemia in 2011. Geneva: World Health Organization; 2015.

2. Oski FA, Honig AS, Helu B, Howanitz P. Effect of iron therapy on behavior performance in nonanemic, iron-deficient infants. Pediatrics. 1983;60:877-880.

3. Akman M, Cebeci D, Okur V, Angin H, Abali O, Akman AC. The effects of iron deficiency on infants' developmental test performance. Acta Paediatr Oslo Nor. 2004;93(10):1391-1396. https://doi.org/10.1111/j.1651-2227.2004.tb02941.x

4. Beard JL. Iron biology in immune function, muscle metabolism and neuronal functioning. J Nutr. 2001;131:568S-580S. https://doi.org/10.1093/jn/131.2.568S

5. Walter T, Arredondo S, Arevalo M, Stekel A. Effect of iron therapy on phagocytosis and bactericidal activity in neutrophils of iron-deficient infants. Am J Gin Nutr. 1986;44:877882. https://doi.org/10.1093/ajcn/44.6.877
6. Yetgin S, Altay C, Ciliv G, et al. Myeloperoxidase activity and bactericidal function of PMN in iron deficiency. Acta Haematol. 1979;61:10-14. https://doi.org/10.1159/000207620

7. Kulapongs $P$, Suskind R, Vithayasi V, et al. Cell mediated immunity and phagocytosis and killing function in children with severe iron deficiency anemia. Lancet. 1974;21:680-691. https://doi.org/10.1016/S0140-6736(74)93264-4

8. Ziegler-Heitbrock L, Hofer TPJ. Toward a refined definition of monocyte subsets. Front Immunol. 2013;4:23. https://doi. org/10.3389/fimmu.2013.00023

9. Ziegler-Heitbrock L. Monocyte subsets in man and other species. Cell Immunol. 2014;289:135-139. https://doi. org/10.1016/j.cellimm.2014.03.019

10. Woollard KJ, Geissmann F. Monocytes in atherosclerosis: subsets and functions. Nature Rev Cardiol. 2010;7:77-86. https:// doi.org/10.1038/nrcardio.2009.228

11. Galkina E, Ley K. Immune and inflammatory mechanisms of atherosclerosis. Annu Rev Immunol. 2009;27:165-197. https:// doi.org/10.1146/annurev.immunol.021908.132620

12. Sadeghian MH, Keramati MR, Ayatollahi H, Manavifar L, Enaiati $\mathrm{H}$, Mahmoudi $M$. Serum immunoglobulins in patients with iron deficiency anemia. Indian J Hematol Blood Transfus. 2010;26:45-48. https://doi.org/10.1007/s12288-010-0025-3

13. Ahluwalia N, Sun J, Krause D, Mastro A, Handte G. Immune function is impaired in iron-deficient, homebound, older women. Am J Clin Nutr. 2004;79(3):516-521. https://doi. org/10.1093/ajcn/79.3.516

14. Berrak SG, Angaji M, Turkkan E, Canpolat C, Timur C, Demiralp-Eksioglu EZ. The effects of iron deficiency on neutrophil/monocyte oxidative burst response in children. Blood. 2006;108(11):3722. https://doi.org/10.1182/blood. V108.11.3722.3722

15. Ekiz C, Agaoglu L, Karakas Z, Gurel N, Yalcin I. The effect of iron deficiency anemia on the function of the immune system. Hematol J. 2005;5(7):579-583. https://doi.org/10.1038/ sj.thj.6200574

16. Mukherjee R, Kanti Barman P, Kumar Thatoi P, Tripathy R, Kumar Das B, Ravindran B. Non-classical monocytes display inflammatory features: validation in sepsis and systemic lupus erythematous. Sci Rep. 2015;5(1):13886. https://doi. org $/ 10.1038 /$ srep13886

17. Wong KL, Tai JJ-Y, Wong WC, Han H, Sem X, Yeap WH, et al. Gene expression profiling reveals the defining features of the classical, intermediate, and nonclassical human monocyte subsets. Blood. 2011;118:16-31. https://doi.org/10.1182/ blood-2010-12-326355

18. Acosta-Rodriguez EV, Rivino L, Geginat J, Jarrossay D, Gattorno M, Lanzavecchia A, et al. Surface phenotype and antigenic specificity of human interleukin 17-producing $T$ helper memory cells. Nat Immunol. 2007;8:639-646. https:// doi.org/10.1038/ni1467

19. Eljaszewicz A, Kleina K, Grubczak K, Radzikowska U, Zembko P, Kaczmarczyk P, et al. Elevated numbers of circulating very small embryonic-like stem cells (VSELs) and intermediate CD14++CD16+ monocytes in IgA nephropathy. Stem Cell Rev Rep. 2018;14(5):686-693. https://doi.org/10.1007/ s12015-018-9840-y

20. Ren X, Mou W, Su C, Chen X, Zhang H, Cao B, et al. Increase in peripheral blood intermediate monocytes is associated with the development of recent-onset type 1 diabetes mellitus in children. Int J Biol Sci. 2017;13(2):209-218. https://doi. org/10.7150/ijbs.15659

21. Oppenheimer SJ. Iron and its relation to immunity and infectious disease. J Nutr. 2001;131(2):616S-635S. https://doi. org $/ 10.1093 / \mathrm{jn} / 131.2 .616 \mathrm{~S}$ 